\title{
Consumption of bonito extract suppresses the decrease in cerebral blood flow in stroke-prone spontaneously hypertensive rats
}

\author{
Masashi Honda ${ }^{1}$, Haruka Yamada ${ }^{2}$, Yoshizu Nozawa ${ }^{1}$, Taichi Ishizaki ${ }^{1}$, Motonaka Kuroda ${ }^{1}$, and Takanori \\ NoGuCHI $^{3}$ \\ ${ }^{1}$ Research Institute for Health Fundamentals, Ajinomoto Co., Inc., Kawasaki; ${ }^{2}$ Faculty of Nutrition, Kobe Gakuin University, Kobe; and \\ ${ }^{3}$ Department of Nutrition and Health Science, Fukuoka Women's University, Fukuoka, Japan
}

(Received 5 June 2010; and accepted 12 July 2010)

\begin{abstract}
The effect of consuming bonito extract (BE) on cerebral blood flow was evaluated in stroke-prone spontaneously hypertensive rats (SHRSP), a cerebrovascular disease model. BE dissolved in drinking water was given to 5-week-old male SHRSP for 7 weeks. Tap water was given to the control group. At the age of 12 weeks, blood flow and vascular diameter were measured in the middle cerebral artery. Both cerebral blood flow and cerebral vessel width were greater in the BE group than in the control group. Also, stroke occurred in 7 (with death in 2) of the 8 animals in the control group but in none of the 6 animals in the BE group. To clarify its mechanism, the expressions of nitrogen oxide synthase (NOS) and the superoxide dismutase activity (SOD) in the brain were evaluated. NOS mRNA expression and SOD activity in the cerebrum were higher in the BE group. These results suggest that the consumption of BE suppresses the decrease of cerebral blood flow and reduces the risk of stroke to maintain vasorelaxation through the production of nitrogen oxide and suppression of active oxygen generation.
\end{abstract}

The rates of diabetes, hypertension, hyperlipidemia, and obesity are increasing due to lifestyle deterioration, such as excessive energy intake and insufficient exercise. Stroke is a serious condition caused by these lifestyle-related diseases, and is likely to leave sequelae, such as paralysis and speech disorders, causing marked impairment of the quality of life $(\mathrm{QOL})(28,29)$; therefore, protection of the cerebral vessels is very important to maintain the QOL.

Stroke-prone spontaneously hypertensive rats (SHRSP) are a strain established by the selective breeding of rats that developed stroke $(24,25)$. SHRSP develop severe hypertension with a systolic

Address correspondence to: Taichi Ishizaki, $\mathrm{PhD}$ Research Institute for Health Fundamentals, Ajinomoto Co., Inc., 1-1 Suzuki-cho, Kawasaki-ku, Kawasaki-shi 210-8681, Japan Tel: +81-44-244-8678, Fax: +81-44-244-9617

E-mail: taichi_ishizaki@ajinomoto.com blood pressure (SBP) exceeding $240 \mathrm{mmHg}$, and suffer stroke accompanied by neurological symptoms, such as convulsion and paralysis similarly to humans. Histopathological changes in SHRSP have been reported to resemble those in humans (34), and the strain is widely used as a hypertensive stroke model. In addition, as the increase in blood pressure is accompanied by a decrease in cerebral blood flow, this model is also used in research related to cerebrovascular diseases $(6,7,13,17,18)$.

Previously we have reported the effect of bonito extract (BE) on peripheral circulation. In peripheral blood flow-lowering mice induced by over-crowding stress, administration of $\mathrm{BE}$ increased peripheral blood flow (8). Also, a single administration of BE and continuous $\mathrm{BE}$ consumption for 4 weeks improved peripheral blood flow in human trials (21, 22). These findings showed the improvement of peripheral blood flow by BE, but the effect on cerebral blood flow was unknown. In this study, we evaluated the effects of BE consumption on cerebral blood 
flow in SHRSP, a cerebrovascular disease model. Also, to clarify its mechanism, we examined the expression of mRNA of nitrogen oxide synthase (NOS), which was related to vasorelaxation and the activity of superoxide dismutase (SOD), an enzyme involved in the elimination of oxidative stressinduced vascular endothelial cell damage (23).

\section{MATERIALS AND METHODS}

Administration sample. Frozen bonito was used as the material for BE. After removing the head and viscera, the bonito bodies were boiled at $95-100^{\circ} \mathrm{C}$ for $1 \mathrm{~h}$. The broth was condensed after separating the oil, and used as BE. Before administration, BE was stored at $-25^{\circ} \mathrm{C}$, and was administered after adjusting its concentration with water. Table 1 shows the nutritional composition of $\mathrm{BE}: 41 \%$ protein or unknown peptides, $22 \%$ free amino acids, $1.2 \%$ peptide, $12 \%$ organic acids, $5 \%$ minerals, and $0.6 \%$ inosinic acid. To measure the total amino acid content, the sample was hydrolyzed by treating it with $6 \mathrm{~N} \mathrm{HCl}$ at $120^{\circ} \mathrm{C}$ for $24 \mathrm{~h}$, and protein and peptides were degraded into free amino acids. The free amino acid composition was determined using an amino acid analyzer (Model L-8500; Hitachi Co., Tokyo, Japan). The difference between the total and free amino acid levels corresponds to amino acids derived from protein and peptides. Organic and nucleic acids were assayed by high-performance liquid chromatography (Model L-7000; Hitachi Co.). Minerals contained in ashed $\mathrm{BE}$ were assayed with an atomic absorptiometer (SAS 7500A; Seiko Instruments, Inc., Chiba, Japan).

Experimental animals. Five-week-old male SHRSP/ Izm (Shimizu Laboratory Supplies Co., Ltd., Kyoto, Japan) were used for the experiment. They were housed at room temperature of $23.2 \pm 2{ }^{\circ} \mathrm{C}$ and humidity of $55 \pm 15 \%$ under 12-hour light and dark cycles (light: $7: 00-19: 00$ ), and were given food and water ad libitum. The food was special SP food developed to efficiently cause stroke in SHRSP (Shimizu Laboratory Supplies Co., Ltd.) $(35,36)$.

Experimental design. BE was dissolved in tap water at a concentration of $8 \%(\mathrm{w} / \mathrm{v})$ and administered continually for 7 weeks from the age of 5 to 12 weeks. The animals were examined daily for symptoms related to stroke, such as hemiparesis. In the final week, urine was collected using 24-hour metabolic cages for the measurement of NOx using $\mathrm{NO}_{2} / \mathrm{NO}_{3}$ Assay Kit-CII (Dojindo Laboratories Co.,
Table 1 Nutrient compositions of bonito extract

\begin{tabular}{lc}
\hline Nutrient & $(\mathrm{g} / 100 \mathrm{~g})$ \\
\hline Free amino acids & 21.5 \\
Alanine & 0.8 \\
Arginine & 0.4 \\
Asparagine & 0.3 \\
Carnosine & 0.4 \\
Creatine & 1.3 \\
Cystine & 0.1 \\
Glutamic acid & 0.5 \\
Glycine & 0.3 \\
Histidine & 10.9 \\
Isoleucine & 0.3 \\
Leucine & 0.6 \\
Lysine & 0.8 \\
Methionine & 0.2 \\
Phenylalanine & 0.3 \\
Proline & 0.5 \\
Serine & 0.3 \\
Taurine & 2.7 \\
Threonine & 0.2 \\
Tryptophan & 0.0 \\
Tyrosine & 0.3 \\
Valine & 0.3 \\
Peptide & \\
Anserine & \\
Organic acid & \\
Lactic acid & \\
Minerals & \\
Magnesium & \\
Potasium & \\
Sodium & \\
Nucleic acid & \\
Inosinic acid & \\
Protein or unknown peptides & \\
\hline & \\
\hline
\end{tabular}

Kumamoto, Japan) based on Griess's method (27). At the end of the experimental period, cerebral blood flow and the middle cerebral artery width were measured, and various organs were weighed. The cerebrum was removed and the appearance of the hemorrhage was examined, and the NOS mRNA expression and SOD activity were measured. This study was carried out according to the ethics rules concerning animal experiments at Kobe Gakuin University and the guidelines of the National Institute of Health (USA).

Blood pressure measurements. Blood pressure was measured weekly by the tail cuff method (10) using UR-1000 (Ueda Electric Works, Co., Tokyo, Japan) from 5 to 11 weeks old. The rat was immobilized using a restrainer maintained at $38^{\circ} \mathrm{C}$, the measure- 
ment was repeated 3 times, and the mean value was adopted.

Blood flow measurements. Cerebral blood flow was measured using the laser Doppler blood flow imaging system PeriScan PIMII (PERIMED Co., Sweden) (12). After the animals were anesthetized by intraperitoneal pentobarbital administration $(60 \mathrm{mg} /$ $\mathrm{kg}$ ), they were placed in a prone position, the scalp was incised, the head was fixed on a stereotactic instrument, the cranium was exposed, and cerebral blood flow was measured by scanning the head.

Measurement of cerebral vessel width. After cerebral blood flow measurement, the cranium at the center of the right parietal bone was thinned using the microgrinder HD20 (Urawa Co., Saitama, Japan) until the cerebral vessel became observable. Mineral oil was dripped to avoid drying, the rat was fixed on the stage under the object lens of a microscope, and images of cerebral blood vessels were recorded on a personal computer via a CCD camera. The vascular width and cerebral blood flow were measured in the middle cerebral artery. Blood vessels were classified on the basis of the method of Ley et al. (16).

Cerebral NOS mRNA expression. mRNA extraction and cDNA synthesis were performed applying routine procedures. mRNA of endothelial type NOS (eNOS), neuronal type NOS (nNOS) and inducible type NOS (iNOS) were measured by quantitative PCR employing an ABI PRISM 7500 Sequence Detection System (Life Technologies Co., CA, USA). The primer sequences were (5'-CTCCAAGTATCC ACGGCATAG-3') for $\beta$-actin(F), (5'-AAGCAATGC TGTCACCTTCC-3') for $\beta$-actin(R), (5'-CCTGTGC ATGGATGAATACGAT-3') for eNOS(F), (5'-TGCC AAATGTGCTGGTCA-3') for eNOS(R), (5'-GTTC GCCTTCTCGATGTTGAC-3') for nNOS(R), (5'-C GGACAGGAAGAAGCTTTCAG-3') for nNOS (F), (5'-TTCACAGTCTTGGTGAAAGCG-3') for iNOS(F), and (5'-AGTAGCAAAGAGGACTGTGG CTC-3') for $\operatorname{iNOS(R).~These~data~were~normalized~}$ relative to the expression of the housekeeping gene $\beta$-actin.

Cerebral SOD activity. An appropriate amount (0.1$0.5 \mathrm{~g}$ ) of cerebral tissue was weighed, homogenized with 10 volumes of a homogenization buffer $(10 \mathrm{mM}$ $\mathrm{KH}_{2} \mathrm{PO}_{4} / \mathrm{Na}_{2} \mathrm{~B}_{4} \mathrm{O}_{7}$ ) using a polytron, the homogenate was sonicated ( $5 \mathrm{~s}, 3$ times), centrifuged $(3,000 \mathrm{rpm}$, $20 \mathrm{~min}$ ), and the supernatant was sampled for the assay. SOD activity was determined by the nitrite method based on Oyagi's method (26).

Statistical analyses. In statistical analyses in the present study, because of 2 deaths in the control group in the final week (12 weeks old), the values for the 6 surviving animals were adopted. All results are expressed as the mean \pm standard error (SE). After variance homogeneity was examined by the Levene test, the $t$-test or Welch's test was performed. Using statistical software SPSS 13.0J (SPSS Japan Inc., Tokyo, Japan), two-tailed tests were performed at the $5 \%$ level of significance.

\section{RESULTS}

\section{Stroke incident and hemorrhage in the brain}

The animals were considered to have developed stroke when abnormal behavior, such as hemiparesis, was observed during the care period. Stroke was observed in 7 (with death in 2) of the 8 animals in the control group but in none (with no deaths) of the 6 animals in the BE group. Hemorrhage (black spots) on the brain surface was found in 4 of the 8 animals in the control group but not in any animal in the BE group.

\section{Body weights during the experimental period}

From the start of the experiment to 11 weeks old, the body weight in both groups increased slightly. The body weight in the control group decreased markedly at 12 weeks old, possibly due to stroke

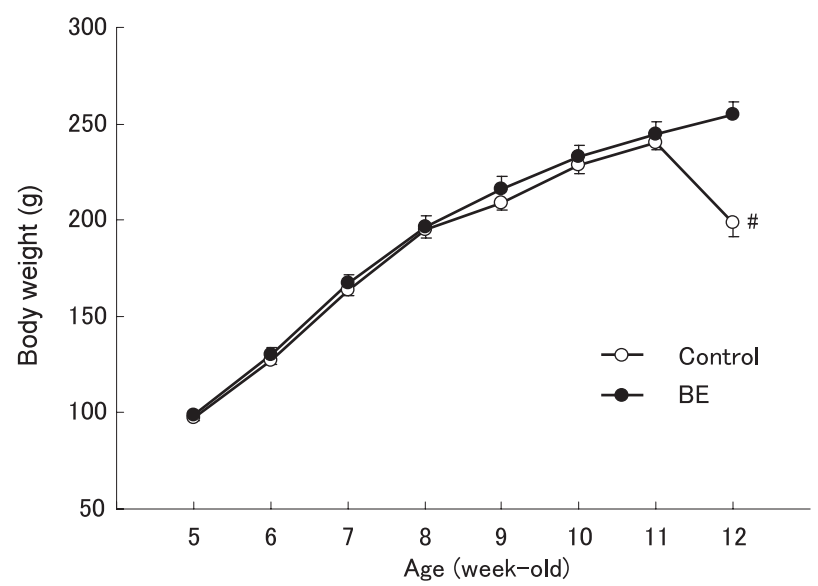

Fig. 1 Changes of body weight during experimental period. The experiment started at 5 weeks old and ended at 12 weeks old. Values are the mean \pm standard error. Control: open circle $(n=8)$, BE: closed circle $(n=6)$. The body weight of $\mathrm{BE}$ (bonito extract) group at 12 weeks old was higher than that of the control group $(\#: P<0.1)$. 
and was lower than the BE group $(P<0.1)$ (Fig. 1).

Intake of feed, drinking volume and energy

Feed intake was significantly lower in the BE than in the control group at $6,7,8,9$, and 10 weeks old, but feed intake in the control group decreased markedly from 11 to 12 weeks old. Drinking water intake was significantly higher in the BE than in the control group at 10,11 , and 12 weeks old. The energy contents of the drinking water containing $\mathrm{BE}$ and feed were $0.23 \mathrm{kcal} / \mathrm{mL}$ and $4.14 \mathrm{kcal} / \mathrm{g}$, respectively. The mean energy intake was significantly higher in the $\mathrm{BE}$ than in the control group at 5,6 , 11, and 12 weeks old, but the mean energy intake in the control group decreased markedly from 11 to 12 weeks old (Fig. 2).

Blood pressure during the experimental period In both groups, both systolic blood pressure (SBP) and diastolic blood pressure (DBP) had increased significantly by the end compared with the start of the experiment $(P<0.01)$. BE suppressed the elevation of blood pressure in both diastole and systole. SBP at 10 and 11 weeks old was significantly lower in the BE than in the control group. DBP at 11 weeks old was significantly lower in the BE than in the control group (Fig. 3).
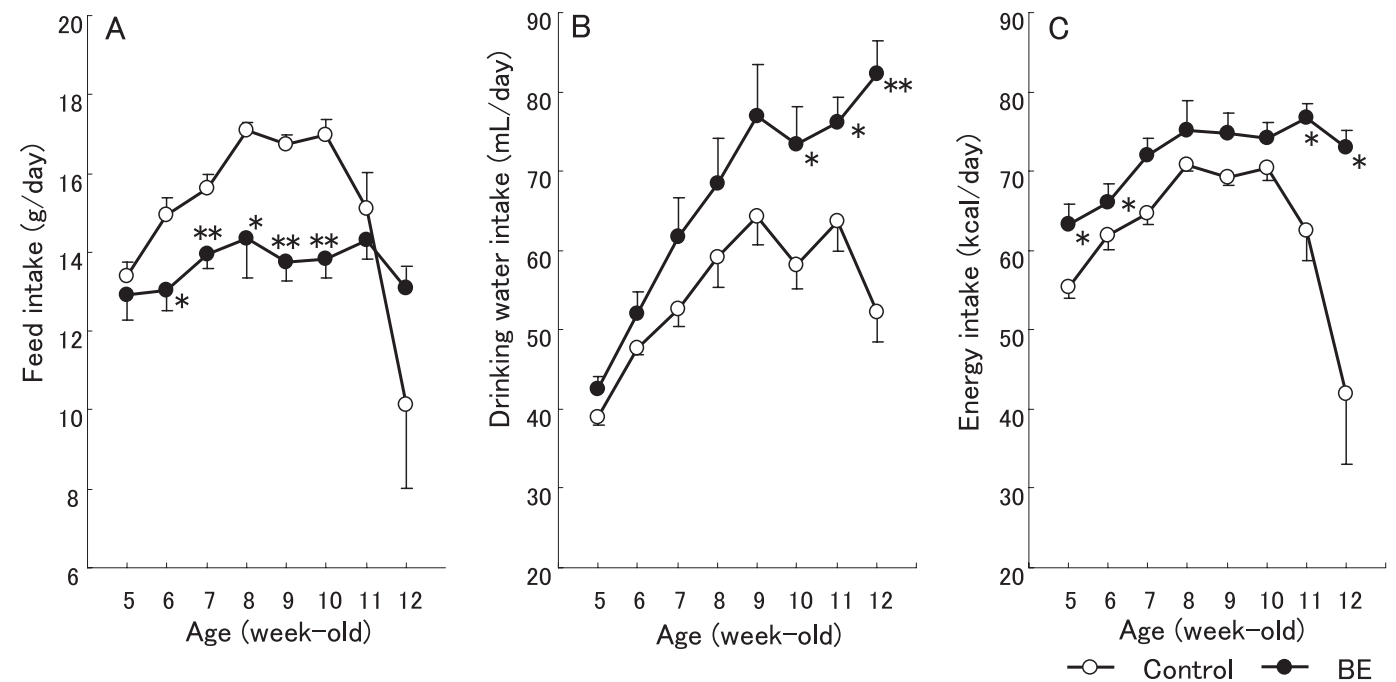

Fig. 2 Changes in feed intake (A), drinking water $(B)$, and energy intake $(C)$ during the experimental period. The experiment started at 5 weeks old and ended at 12 weeks old. Values are the mean \pm standard error. Control: open circle $(n=8)$, BE (bonito extract): closed circle $(n=6)$. Significant difference compared with the control group $\left(*: P<0.05,{ }^{* *}: P<0.01\right)$.
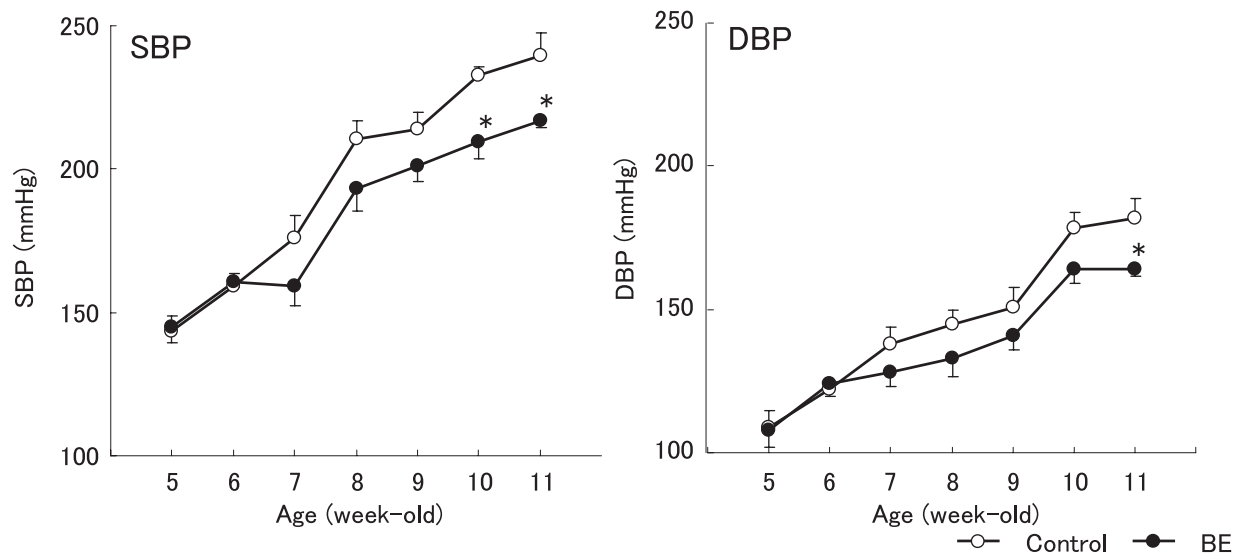

Fig. 3 Changes of systolic blood pressure (SBP) and diastolic blood pressure (DBP) during the experimental period. Blood pressure was measured weekly from 5 to 11 weeks old. Values are the mean \pm standard error. Control: open circle $(n=8)$, BE (bonito extract): closed circle $(n=6)$. Significant difference compared with the control group $\left({ }^{*}: P<0.05,{ }^{* *}: P<0.01\right)$. 
Organ weights at the end of experiment

The kidney and liver weights were significantly higher, but the heart and brain weights were significantly lower in the BE than in the control group (Table 2).

Cerebral blood flow and cerebral vessel width Cerebral blood flow was significantly higher in the

Table 2 Organ weights at the end of the experiment (g/100g body weight)

\begin{tabular}{ccc}
\hline Organ & Control & \multicolumn{1}{c}{ BE } \\
\hline Kidney & $0.43 \pm 0.01$ & $0.51 \pm 0.01^{* *}$ \\
Liver & $3.48 \pm 0.11$ & $4.04 \pm 0.13^{* *}$ \\
Heart & $0.53 \pm 0.01$ & $0.48 \pm 0.01^{*}$ \\
Brain & $0.83 \pm 0.03$ & $0.71 \pm 0.02^{* *}$ \\
\hline
\end{tabular}

Values are the mean \pm standard error $(n=6)$.

Significant differences compared with the control group (*: $P<$ $0.05, * *: P<0.01)$. BE: bonito extract

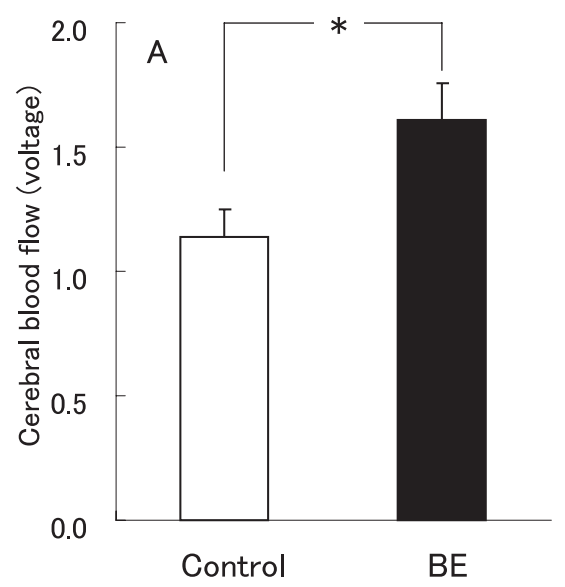

$\mathrm{BE}$ than in the control group. Cerebral vascular width was significantly greater in the $\mathrm{BE}$ than in the control group (Fig. 4).

\section{Biochemical parameters}

In three isoforms of NOS, iNOS mRNA showed obvious differences between the BE and control groups. The expression of eNOS mRNA in the cerebrum was higher $(P<0.1)$ in the BE than in the control group. The expression of iNOS mRNA in the cerebrum was significantly higher in the BE than in the control group. The expression of nNOS mRNA in the cerebrum showed no difference between groups. Cerebral SOD activity was higher $(P$ $<0.1)$ in the BE than in the control group. The urinary level of NOx, the end-metabolite of nitrogen oxide, was also higher $(P<0.1)$ in the $\mathrm{BE}$ than in the control group (Table 3$)$. 


\section{DISCUSSION}

SHRSP are characterized by a spontaneous decrease in blood flow and the development of hypertension, with the frequent presentation of stroke (cerebral infarction and cerebral hemorrhage) with aging due to a genetic mechanism, and are widely used as a model animal for research into blood flow $(6,7,13$, $17,18)$. In this study, using this strain, we evaluated the effects of BE consumption on the cerebral circulation and its mechanism by measuring the nitrogen oxide level and biochemical parameters related to active oxygen.

A marked decrease of body weight in the control group accompanied by decreased energy intake was observed at the end of the experiment. The incidence of stroke in the control group in the final week explains the feed intake decrease. Indeed, two individuals died in the final week (12 weeks old). From the start of the experiment (5 weeks old) to 10 weeks old, drinking water intake was significantly higher, but feed intake was lower in the BE than in the control group. Regarding the reason for these results, rats have been reported to show a preference for BE (14) and to have drunk it willingly and reduced their feed intake as if to compensate. Kasaoka et al. (11) fed rats feed supplemented with a BE concentrate and a high histidine content, and reported decreased food intake and fat accumulation. In the present study, the same dietary behavior might have been observed before stroke in the control group.

We previously reported a clinical study using elderly subjects in which SBP was reduced by the consumption of BE (33). A similar improvement in blood pressure was also observed in this study using SHRSP, a hypertensive model animal. Both SBP and DBP in the BE group were significantly lower than in the control group at the end of the experiment, and a difference in organ weights related to the improvement of blood pressure was observed. SHRSP are known to develop glomerular disorders with growth (19). The kidney weight was higher in the $\mathrm{BE}$ than in the control group, and the urinary protein level was lower in the $\mathrm{BE}$ than in the control group in the final week (control: $306.1 \pm 45.6 \mathrm{mg}$ / $\mathrm{dL}, \mathrm{BE}: 48.4 \pm 5.5 \mathrm{mg} / \mathrm{dL}$ ). These data suggested that $\mathrm{BE}$ consumption might prevent renal disorder of SHRSP with growth. Heart weight was lower in the $\mathrm{BE}$ than in the control group, suggesting that BE intake suppressed heart hypertrophy induced by blood pressure elevation.

Cerebral blood flow was significantly higher in the BE than in the control group. Also, while the cerebral vascular width narrowed in the control group, this was not the case in the BE group. SHRSP are known to show brain enlargement with growth due to brain edema, etc. (9). In the present study, brain weight was significantly lower in the $\mathrm{BE}$ than in the control group. It is considered that the maintenance of cerebral blood flow by BE prevented brain enlargement; therefore, the frequencies of both stroke and death were lower in the BE than in the control group, and no animal showed cerebral hemorrhage in the BE group. These observations suggest that $\mathrm{BE}$ suppressed the incidence of stroke by preventing the decrease in cerebral blood flow.

Since cerebral blood flow was increased by the administration of BE, we measured the NOS mRNA expression in the cerebrum to evaluate the mechanism of this increase. NOS synthesizes nitrogen oxide from L-arginine in endothelial cells and relaxes vascular smooth muscle cells. NOS has three isoforms: eNOS expressed in vascular endothelial cells, nNOS expressed in nerve cells, and iNOS expressed in immunocytes and vascular smooth muscle cells. In this study, eNOS and iNOS mRNA was expressed more in the BE than in the control group. Administration of statins, HMG-CoA reductase inhibitors, improved cerebral blood flow, but have been reported not to improve cerebral blood flow (3). An experiment on contraction-relaxation responses to the administration of L-arginine and NOS inhibitors has reported that cerebral vessel relaxation responses in SHR are related to the level of iNOS expression (1). These reports indicate the involvement of eNOS and iNOS in cerebral blood flow, and suggest that NOS expression in cerebral vascular endothelial and smooth muscle cells is involved in preventing decreased cerebral blood flow by BE.

There is a report in which disorders of endothelium-dependent vascular relaxation were due to the enhancement of active oxygen synthesis in the endothelium (5). Since the results of this study concerning NOS mRNA and urinary NOx suggested that endothelium-dependent relaxation was maintained, we also measured SOD activity in the cerebrum, and it was higher in the BE than in the control group. Furthermore, intermediates with strong oxidative activity, such as peroxynitrite $\left(\mathrm{ONOO}^{-}\right)$, are known to be produced by the interaction between 2 radicals generated by completely independent mechanisms, i.e., NO and superoxide $\left(\mathrm{O}_{2}{ }^{-}\right)$(4). SOD and NOS were activated by the consumption of $\mathrm{BE}$, which may have suppressed $\mathrm{ONOO}^{-}$production and protected the body from ox- 
idative stress.

Sesamin (20), vitamins C and E (2), ginkgo leaf extract (31), and chlorella (30) have been reported as food materials with preventive effects on decreased cerebral blood flow and the occurrence of stroke in SHRSP. On comparing the effects of these materials with that of BE, blood flow showed a marked variation depending on the measurement device and site; however, according to the literature on SBP, the effect of BE was similar to those of sesamin and chlorella. The amino acids contained in BE include arginine, which serves as a substrate for NOS. Components of BE with antioxidant activity include histidine and anserine (15). Histidine is the most abundant amino acid in $\mathrm{BE}$, anserine is a peptide consisting of beta-alanine and 1-methylhistidine, and both have an imidazole ring. BE also contains melanoidin and aromatic components, and shows antioxidant activity (32). The preventive effects of BE on the cerebrovascular system might be related to the antioxidant effects of these various components, and it was same that preventive effects of sesamin and chlorella suggested to relate anti oxidant effects.

In this study, BE was found to suppress the ageassociated decrease in cerebral blood flow and cerebral vessel atrophy in SHRSP and to reduce the risk of cerebrovascular disorders, such as stroke. These results suggest the effectiveness of $\mathrm{BE}$ to protect the QOL.

\section{REFERENCES}

1. Briones AM, Alonso MJ, Marin J and Salaices M (1999) Role of iNOS in the vasodilator responses induced by Larginine in the middle cerebral artery from normotensive and hypertensive rats. Br J Pharmacol 126, 111-120.

2. Chen X, Touyz RM, Park JB and Schiffrin EL (2001) Antioxidant effects of vitamins $\mathrm{C}$ and $\mathrm{E}$ are associated with altered activation of vascular NADPH oxidase and superoxide dismutase in stroke-prone SHR. Hypertension 38, 606-611.

3. Endres M, Laufs U, Huang Z, Nakamura T, Huang P, Moskowitz MA and Liao JK (1998) Stroke protection by 3-hydroxy-3-methylglutaryl (HMG)-CoA reductase inhibitors mediated by endothelial nitric oxide synthase. Proc Natl Acad Sci USA 95, 8880-8885.

4. Fujii J and Taniguchi N (1995) Cross talk between NOS and SOD. Experimental Medicine 13, 984-989. (in Japanese)

5. Grunfeld S, Hamilton CA, Mesaros S, McClain SW, Dominiczak AF, Bohr DF and Malinski T (1995) Role of superoxide in the depressed nitric oxide production by the endothelium of genetically hypertensive rats. Hypertension 26, 854-857.

6. Higashino H, Simeonova K, Lambev I and Suzuki A (1997) Tissue variation of acute haemodynamic changes by NGnitro-L-arginine in stroke-prone spontaneously hypertensive and Wistar-Kyoto rats. Clin Exp Pharmacol Physiol 24, 249-
255.

7. Higashino H, Suzuki A, Simeonova KD and Lambev IV (1997) Thermo-elevation effect on blood flow in stroke-prone spotaneously hypertensive rats (SHRSP). Acta Med Kinki Univ 22, 209-215.

8. Honda M, Nozawa Y, Ishizaki T and Kuroda M (2009) Ingestion of bonito extract ameliorates peripheral blood flow in mice loaded from over crowding stress. Biomed Res 30, 129 135.

9. Ikeda K, Nara Y, Matumoto C, Mashimo T, Tamada T, Sawamura M, Nabika T and Yamori Y (1996) The region responsible for stroke on chromosome 4 in the stroke-prone spontaneously hypertensive rat. Biochem Biophys Res Commun 229, 658-662.

10. Ikeda K, Nara Y and Yamori Y (1991) Indirect systoric and mean blood pressure determination by a new tail cuff method in spotaneously hypertensive rats. Lav Anim 25, 26-29.

11. Kasaoka S, Goto G, Tokitaka M, Takizawa M, Tanaka K, Ogawa M and Nakajima S (2008) Effect of a bonito bouillon extract on food intake and fat accumulation in rats. Jpn $J$ Nutr Diet 66, 127-132. (in Japanese with English abstract)

12. Kashima S (1993) Non-contact laser tissue blood flowmetry. Jap J Med Instrumentation 63, 301-306. (in Japanese with English abstract)

13. Katayama Y, Suzuki S, Shimizu J, Inamura K, Sugimoto S and Terashi A (1986) Effect of blilateral common carotid artery ligation on prostaglandin levels (TXA2, PGI2) in spontaneously hypertensive rats (SHRSP, SHRSR) and normotensive rats (WKY). Brain Nerve 38, 571-578. (in Japanese)

14. Kawasaki H, Yamada A and Fushiki T (2003) Effect of early flavor experience of bonito bouillon-flavored diet on the flavor preference of adult mice. J Cookery Sci Jap 36, 116122. (in Japanese with English abstract)

15. Kohen R, Yamamoto Y, Cundy KC and Ames BN (1988) Antioxidant activity of carnosine, homocarnosine, and anserine present in muscle and brain. Proc Natl Acad Sci USA 85, 3175-3179.

16. Ley K, Pries AR and Gaehtgens P (1986) Topological structure of rat mesenteric microvessel networks. Microvasc Res 32, 315-332.

17. Nagao T, Sadoshima S, Ishitsuka T, Kusuda K, Shiokawa O, Ibayashi S and Fujishima M (1992) Effects of acute superior cervical ganglionectomy on cerebral blood flow and metabolism in stroke-prone spontaneously hypertensive rats subjected to cerebral ischaemia. Clin Exp Pharmacol Physiol 19, 489-493.

18. Nagisa Y (2008) Vascular complication models using strokeprone spontaneously hypertensive rats. Folia Pharmacol Jpn 132, 207-212. (in Japanese)

19. Nakamura T, Obata J, Kuroyanagi R, Kimura H, Ikeda Y, Takano H, Naito A, Sato T and Yoshida Y (1996) Involvement of angiotensin II in glomeruloscrelosis of stroke-prone spotaneously hypertensive rats. Kidney Int 49, S109-S112.

20. Noguchi T, Ikeda K, Sasaki Y, Yamamoto J, Seki J, Yamagata K, Nara Y, Hara H, Kakuta H and Yamori Y (2001) Effects of vitamin $\mathrm{E}$ and sesamin on hypertension and cerebral thrombogenesis in stroke-prone spontaneously hypertensive rats. Hypertens Res 24, 735-742.

21. Nozawa Y, Ishizaki T, Kuroda M and Noguchi T (2008) Effect of dried-bonito broth intake on peripheral blood flow, mood, and oxidative stress maker in humans. Physiol Behav 93, 267-273.

22. Nozawa Y, Kuroda M and Noguchi T (2007) Consumption 
of dried-bonito broth acutely increases peripheral blood flow in humans. J Health Sci 53, 339-343.

23. Nozik-Grayck E, Suliman HB and Piantadosi CA (2005) Extracellular superoxide dismutase. Int J Biochem Cell Biol 37, 2466-2471.

24. Okamoto K and Aoki K (1963) Development of a strain of spontaneously hypertensive rats. Jpn Circ J 27, 282-293.

25. Okamoto K, Yamori Y and Nagaoka A (1974) Eatablishment of the stroke-prone spontaneously hypertensive rat (SHR). Cir Res 34-35, 143-153.

26. Oyanagi Y (1984) Establishment of nitrite-kit for SOD activity determination. Jap J Inflammation 4, 63-73. (in Japanese)

27. Pollack JS, Forstermann U, Mitchell JA, Warner TD, Schmid HHWS, Nakane M and Murad M (1991) Purification and characterization of particulate endothelium-derived relaxing factor synthase from cultured and native bovine aortic endotherial cells. Proc Natl Acad Sci USA 88, 10480-10484.

28. Saeki S, Chisaka H and Hachisuka K (2005) Life satisfaction and functional disabilities in long-term survivors after first stroke. J UOEH 27, 171-177.

29. Saeki S and Hachisuka K (2006) Long-term prognosis of stroke patients after medical rehabilitation. J Clin Rehabil 15, 818-823. (in Japanese)

30. Sansawa H, Takahashi M, Tsuchikawa S and Endo H (2006) Effect of chlorella and its fractions on blood pressure, cerebral stroke resions, and life span in stroke-prone spotaneous- ly hypertensive rats. J Nutr Sci Vitaminol 52, 457-466.

31. Sasaki Y, Noguchi T, Yamamoto E, Giddings JC, Ikeda K, Yamori Y and Yamamoto J (2002) Effects of Ginkgo biloba extract (EGB 761) on cerebral thrombosis and blood pressure in stroke-prone spontaneously hypertensive rats. Clin Exp Pharmacol Physiol 29, 963-967.

32. Suzuki T and Motosugi M (1991) Antioxydative activity of broiled-dryed skipjack(Katsuobushi) extracts. J Jpn Soc Food Sci Tech 38, 675-680. (in Japanese with English abstract)

33. Umeki Y, Hayabuchi H, Hisano M, Kuroda M, Honda M, Ando B, Ohta M and Ikeda M (2008) The effect of the dried-bonito broth on blood pressure, 8-hydroxydeoxyguanosine (8-OHdG), an oxidative stress marker, and emotional states in elderly subjects. $J$ Clin Biochem Nutr 43, 175-184.

34. Yamori Y, Horie R, Handa H, Sato M and Fukase M (1976) Pathogenetic similarity of strokes in stroke-prone spontaneously hypertensive rats and humans. Stroke 7, 46-53.

35. Yamori Y, Horie R, Tanase H, Fujiwara K, Nara Y and Lovenberg W (1984) Possible role of nutritional factors in the incidence of cerebral lesions in stroke-prone spontaneously hypertensive rats. Hypertension 6, 49-53.

36. Yamori Y, Nara Y, Tsubouchi T, Sogawa Y, Ikeda K and Horie $\mathrm{R}$ (1986) Dietary prevention of stroke and its mechanisms in stroke-prone spontaneously hypertensive rats-preventive effect of dietary fibre and palmitoleic acid. J Hypertens Suppl 4, S449-452. 